\title{
Production and study of heavy neutron rich nuclei formed in multi-nucleon transfer reactions
}

\section{I. Zagrebaev, S. G. Zemlyanoy, E. M. Kozulin, Yu. Kudryavtsev, V. Fedosseev, R. Bark \& H. A. Othman}

\section{Hyperfine Interactions}

ISSN 0304-3843

Volume 216

Combined 1-3

Hyperfine Interact (2013) 216:109-113

DOI 10.1007/s10751-013-0838-1
VOLUME 216

Nos. 1-3

ISSN 034043843

CODEN HYINDN

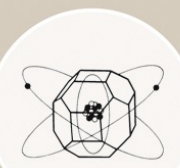

JOURNAL DEVOTED TO RESEARCH IN THE BORDER REGIONS OF SOLID STATE, ATOMIC AND NUCLEAR PHYSICS

\section{HYPERFINE INTERACTIONS}

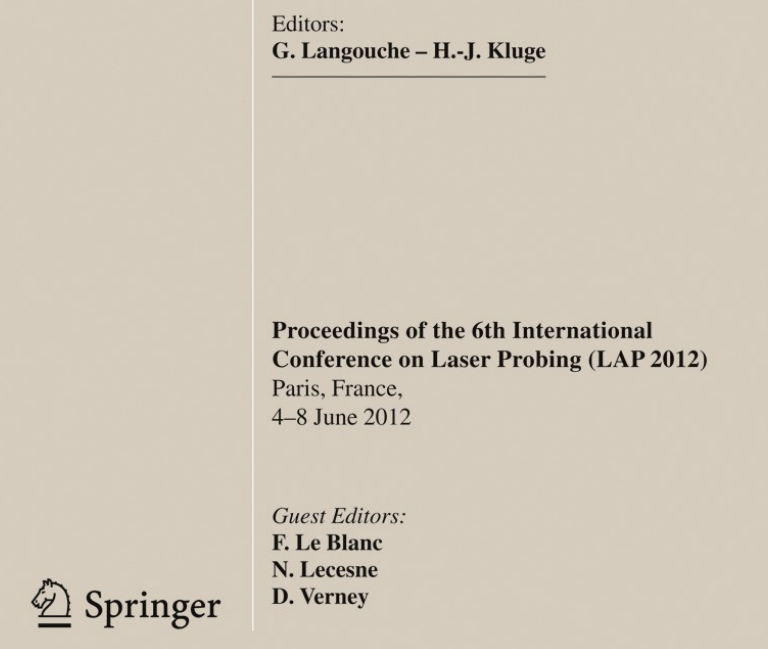

黛 Springer 
Your article is protected by copyright and all rights are held exclusively by Springer Science +Business Media Dordrecht. This e-offprint is for personal use only and shall not be selfarchived in electronic repositories. If you wish to self-archive your article, please use the accepted manuscript version for posting on your own website. You may further deposit the accepted manuscript version in any repository, provided it is only made publicly available 12 months after official publication or later and provided acknowledgement is given to the original source of publication and a link is inserted to the published article on Springer's website. The link must be accompanied by the following text: "The final publication is available at link.springer.com". 


\title{
Production and study of heavy neutron rich nuclei formed in multi-nucleon transfer reactions
}

\author{
V. I. Zagrebaev • S. G. Zemlyanoy • E. M. Kozulin • \\ Yu. Kudryavtsev • V. Fedosseev • R. Bark • H. A. Othman
}

Published online: 13 March 2013

(C) Springer Science+Business Media Dordrecht 2013

\begin{abstract}
A new setup is proposed to produce and investigate heavy neutron-rich nuclei located along the neutron closed shell $\mathrm{N}=126$. This "blank spot" of the nuclear map can be reached neither in fusion-fission reactions nor in fragmentation processes widely used nowadays for the production of exotic nuclei. The present limits of the upper part of the nuclear map are very close to stability while the unexplored area of heavy neutron-rich nuclides along the neutron closed shell $\mathrm{N}=$ 126 is extremely important for nuclear astrophysics investigations and, in particular, for the understanding of the r-process of astrophysical nucleosynthesis. A new way was recently proposed for the production of these nuclei via low-energy multinucleon transfer reactions. The estimated yields of neutron-rich nuclei are found to be rather high in such reactions and several tens of new nuclides can be produced, for example, in the near-barrier collision of $136 \mathrm{Xe}$ with $208 \mathrm{~Pb}$. This setup could definitely open a new opportunity in the studies at heavy-ion facilities and will have significant impact on future experiments.
\end{abstract}

Proceedings of the 6th International Conference on Laser Probing (LAP 2012), Paris, France, 4-8 June 2012.

V. I. Zagrebaev · S. G. Zemlyanoy $(\bowtie) \cdot$ E. M. Kozulin

FLNR, Joint Institute for Nuclear Research, 141980 Dubna, Russia

e-mail: zemlya@jinr.ru

Yu. Kudryavtsev

Instituut voor Kern-en Stralingsfysica, K.U. Leuven, 3001 Leuven, Belgium

V. Fedosseev

CERN, 1211, Geneva 23, Switzerland

R. Bark

iThemba LABS, Nat. Research Foundation, Somerset West 7129, South Africa

H. A. Othman

Physics Department, Faculty of Science, Menoufiya University,

Shibin El-kom, Menoufiya, Egypt 
Keywords Heavy neutron-rich nuclei • r-process • Multi-nucleon transfer reactions • Gas cell $\cdot$ Laser ionization $\cdot$ Mass-separation

\section{Physics objectives}

Properties of light and medium mass nuclei located far from the stability line have been studied already for many years. As a rule, the light exotic nuclei are produced in fragmentation processes and the medium mass neutron rich nuclei with $A \sim 100$ in fision reactions. Production and study of heavier neutron rich nuclei with $A>$ 160 encounters both physical and technical problems. Due to the "curvature" of the stability line (its increasing bending to the neutron axis), fusion reactions of stable nuclei produce only proton rich isotopes of heavy elements. For example, in the fusion of rather neutron rich ${ }^{18} \mathrm{O}$ and ${ }^{186} \mathrm{~W}$ isotopes one may get only the neutron deficient ${ }^{204} \mathrm{~Pb}$ excited compound nucleus, which after evaporation of several neutrons shifts even more to the proton rich side. As a result, on the nuclear map there are, for example, 19 known neutron rich isotopes of cesium $(Z=55)$ and only 4 of platinum $(Z=78)$. For elements with $Z>100$ only neutron deficient isotopes (located to the left of the stability line) have been synthesized so far. That is the main reason also for the impossibility to reach the center of the "island of stability" ( $\mathrm{Z} \sim 114$ and $\mathrm{N} \sim 184)$ in the superheavy mass region, and neutron deficient isotopes of elements with $Z>120$ (being synthesized in fusion reactions) should have very short half-lives (less than one microsecond), insufficient for their separation and identification.

At the same time, the unexplored area of heavy neutron rich nuclei is extremely important for nuclear astrophysics investigations and, in particular, for the understanding of the r-process of astrophysical nucleogenesis (a sequence of neutroncapture and $\beta^{-}$-decay processes). Just in this region the closed neutron shell $N=126$ is located which is the last "waiting point" in the r-process (see Fig. 1). The half-lives and other characteristics of these nuclei feed directly into the r-process scenario of supernovae explosions. Study of the structural properties of nuclei along the neutron shell $N=126$ could also contribute to the present discussion of the quenching of shell gaps in nuclei with large neutron excess.

There are three possibilities for the production of such nuclei. These are the multi-nucleon transfer reactions [1], fusion reactions with extremely neutron rich radioactive nuclei and rapid neutron capture processes. Today the two last methods look unrealizable because of low intensity of radioactive beams and low neutron fluxes in existing nuclear reactors. On the contrary, the low-energy multi-nucleon transfer reactions, as well as the quasi-fission processes [2] (which are similar) are quite practical. They can be used for the production of new neutron rich isotopes not only in the region of $\mathrm{Z} \sim 80$ but also in the superheavy mass area [3]. Theoretical estimations show that several tens of new nuclides in the region of $N=126$ and $\mathrm{Z} \sim 75$ can be produced, for example, in the near-barrier collision of ${ }^{136} \mathrm{Xe}$ with ${ }^{208} \mathrm{~Pb}$ (see Fig. 2).

Neutron rich isotopes of transfermium elements can also be produced in the multinucleon transfer reactions at low-energy collisions of actinide nuclei. Production and study of properties of neutron rich fermium isotopes $(A>260)$ are extremely interesting for several reasons. Firstly, as mentioned above, all known isotopes of 


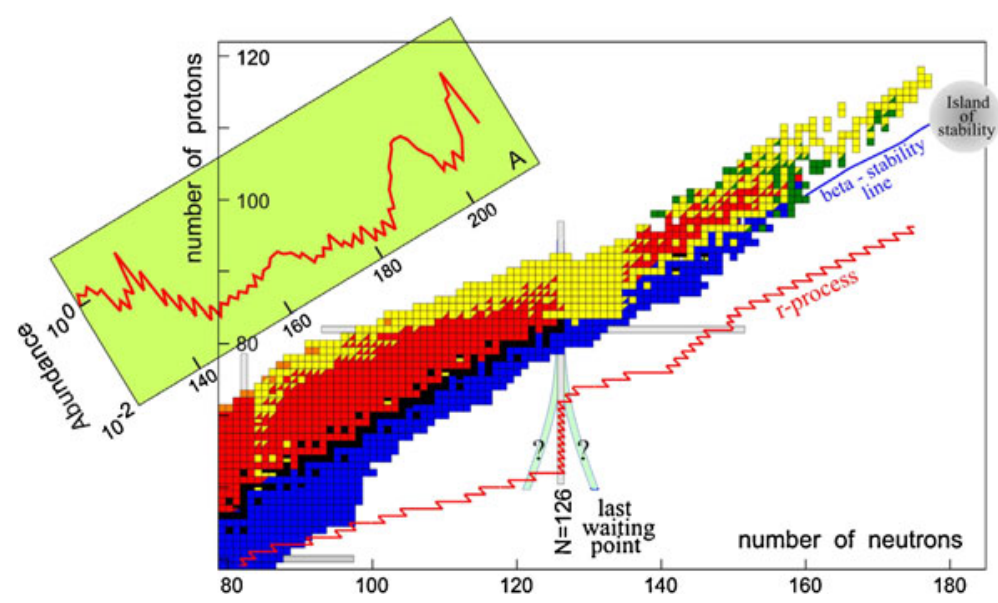

Fig. 1 Upper part of the nuclear map. r-process of nucleosynthesis is shown schematically
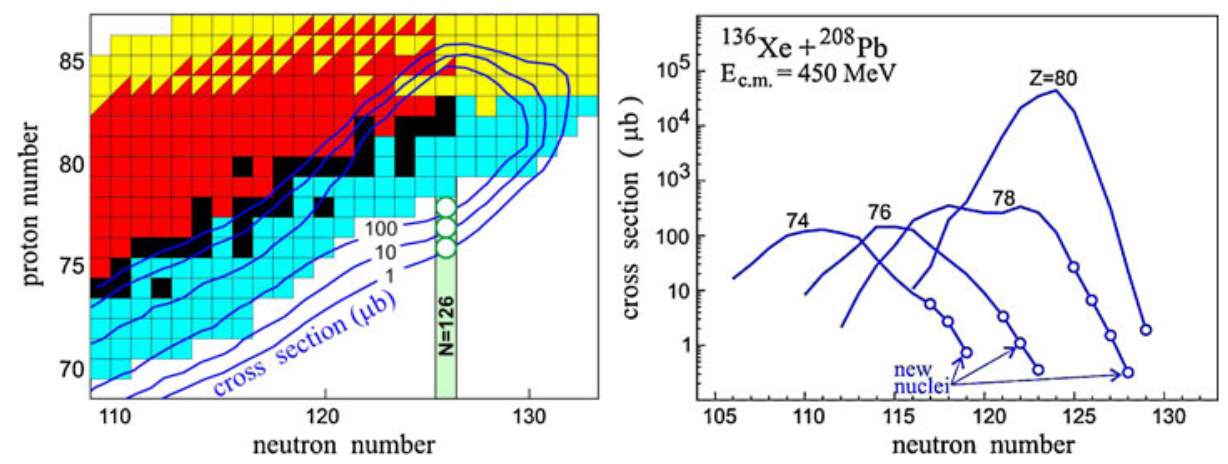

Fig. 2 Cross sections for formation of heavy nuclei in collisions of $136 \mathrm{Xe}+208 \mathrm{~Pb}$ at center-of-mass energy of $450 \mathrm{MeV}$ [1]. Open circles in the right panel correspond to unknown isotopes

fermium (and of more heavy elements) are located to the left side of the beta-stability line (see Fig. 1). Secondly, the well known "fermium gap" (isotopes ${ }^{258-260} \mathrm{Fm}$ with very short half-lives for spontaneous fission) impedes formation of nuclei with $Z>100$ by the weak neutron fluxes realized in existing nuclear reactors. In this connection, it is extremely interesting to know what is the first $\beta^{-}$-decaying fermium isotope and how long is its half-life. This is important not only for reactor-based physics but also for explosive nucleosynthesis in which this fermium gap might be bypassed.

Unfortunately, the neutron rich heavy nuclei with $Z>70$ formed in the multinucleon transfer reactions cannot be separated and studied at available setups created quite recently just for studying the products of deep inelastic scattering (such as VAMOS, PRISMA and others). These fragment separators (as well as other setups) cannot separate heavy nuclei with $Z>70$ by their atomic number (mass separation is more simple with time-of-flight technique, for example). 


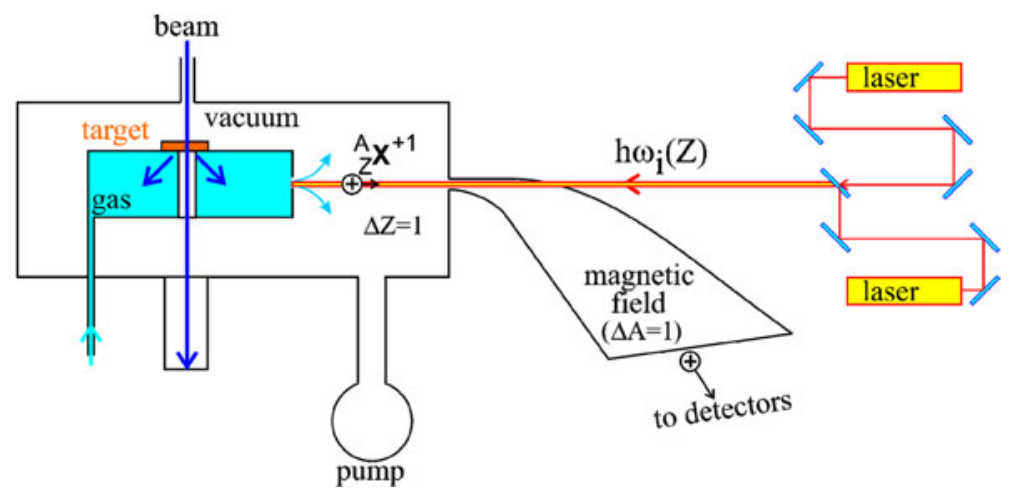

Fig. 3 Schematic view of setup for resonance laser ionization of nuclear reaction products stopped in gas with subsequent mass separation of them and transportation to detecting system

\section{Proposed scheme of setup}

During the last several years a combined method of $\mathrm{Z}$ and $\mathrm{A}$ separation has been intensively studied and developed based on stopping nuclei in gas and subsequent resonance laser ionization [5,6]. This method was used up to now for separation and study of light exotic nuclei and fission fragments. Such techniques allows one to extract nuclei with a given atomic number, while a separation of the single-ionized isotopes by their mass number can be done rather easily by a magnetic field. Halflives of heavy neutron rich nuclei, which we are interested in (as a rule, $\beta^{-}$-decaying), are much longer than the extraction time of ions at such a setup.

The scheme of this setup is shown in Fig. 3. Neutron rich isotopes of heavy elements are produced in multi-nucleon transfer reactions with heavy ions accelerated up to 5-10 MeV/nucleon (depending on projectile-target combination). The target, a foil of about $300 \mu \mathrm{g} / \mathrm{cm}^{2}$ thickness (or larger), is placed at the window of gas cell (or inside it). Nuclear reaction products recoiling from the target as multi-charged ions, are decelerated and neutralized due to collisions with the electrons and atoms of buffer gas in the gas cell filled with pure argon or helium. Then the desired atoms (with a given $\mathrm{Z}$ number) are ionized by means of two or three-step resonance laser irradiation and extracted through the exit hole or supersonic nozzle into the vacuum volume as positive charged ions $(Q=+1)$ with slow energies of about $0.2 \mathrm{eV}$. In vacuum the ions are transported through the sextupole or quadrupole ion-guide system (see below), which helps to pump away the residual buffer gas and keep the ions.

Then the ions are accelerated up to $30-60 \mathrm{keV}$ and separated by the magnet of the mass-separator. A detailed description of the technique of stopping and cooling the ion beams in buffer gas, laser ionization and separation can be found in [4-14]. In this way a low-energy beam of singly-ionized ions is produced with an extremely small emittance. Atomic nuclei of this beam have a definite value of charge number and a given (chosen) mass value, there are neither isobars nor isotopes. This allows one to perform subsequent high sensitive analysis of spectroscopic and decay properties of these nuclei, as well as a measurement of spins, magnetic dipole and electric quadrupole moments and charge radii of these nuclei by means of laser spectroscopy. 
The use of the designed setup for separation and study of heavy neutron rich nuclei does not require any specific beams of accelerated ions. The ion beams available at FLNR fully satisfy our requirements which look as follows: the ionsfrom ${ }^{16,18} \mathrm{O}$ to ${ }^{238} \mathrm{U}$ (i.e., quite different depending on the problem to be solved), beam energies 4.5-9 MeV/nucleon (slightly above the Coulomb barrier), beam intensity up to $10^{13}$ pps with beam size at the target 3-10 mm and beam emittance $20 \pi \mathrm{mm}$ mrad. The different heavy targets, including actinides it is expected to use.

\section{Outlook}

It is evident that the proposed method allows one to extract not only heavy transfer reaction products but also any other nuclei with half-lives longer than a few tens of milliseconds including neutron rich fission fragments, fusion reaction products and light exotic nuclei. Such studies are already performed at the similar setups, for example, in Finland and Belgium, and at some different with a hot cavity facility in CERN. Estimated efficiency of such setups is about $10 \%$ and depends on the half-life or the extracted ion. This is quite sufficient for the study of nuclear reaction products formed with cross sections of about 1 microbarn at a beam intensity of $0.1 \mathrm{p} \mu \mathrm{A}$. This method allows one to study the structure and decay properties of new exotic nuclei as well as spectroscopic properties of the corresponding atoms. At higher beam intensity this setup could be used also for separation and study of neutron rich long living superheavy nuclei produced in multi-nucleon transfer reactions with actinide targets (see above).

\section{References}

1. Zagrebaev, V., Greiner, W.: Phys. Rev. Lett. 101, 122701 (2008)

2. Itkis, M.G., et al.: Nucl. Phys. A734, 136 (2004)

3. Zagrebaev, V.I., Oganessian, Y.T., Itkis, M.G., Greiner, W.: Phys. Rev. C73, 031602(R) (2006)

4. Köster, U., et al.: Spectrochim. Acta B58, 1047 (2003)

5. Kudryavstev, Y., et al.: Nucl. Instrum. Methods Phys. Res. B204, 336 (2003)

6. Moore, I.D., et al.: J. Phys. G: Nucl. Part. Phys. 31, S1499 (2005)

7. Ärje, J., Äystö, J., Hyvönen, H., Taskinen, P., Koponen, V., Honkanen, J., Hautojärvi, A., Vierinen, K.: Phys. Rev. Lett. 54, 99 (1985)

8. Savard, G., et al.: Nucl. Instrum. Methods Phys. Res. B204, 582 (2003)

9. Äystö, J.: Nucl. Phys. A693, 477 (2001)

10. Vermeeren, L., Bijnens, N., Huyse, M., Kudryavtsev, Y.A., Van Duppen, P., Wauters, J., Qamhieh, Z.N., Silverans, R.E., Thoen, P., Vandeweert, E.: Phys. Rev. Lett. 73, 1935 (1994)

11. Kudryavtsev, Y., et al.: Nucl. Instrum. Methods Phys. Res. B114, 350 (1996)

12. Kessler, T., Moore, I., Kudryavtsev, Y., Peräjärvi, K., Popov, A., Ronkanen, P., Sonoda, T., Tordoff, B., Wendt, K., Äystö, J.: Nucl. Instrum. Methods Phys. Res. B266, 681 (2008)

13. Kudryavtsev, Y., et al.: Nucl. Instrum. Methods Phys. Res. B267, 2908 (2009)

14. Ravn, H.L., Allardyce, B.W.: On-line mass separators. In: Allan Bromley, D. (ed.) Treatise on Heavy-Ion Science, vol. 8, p. 363. Plenum Publishing Corporation (1989) 